\title{
Clinical genetics evaluation in identifying the etiology of autism spectrum disorders
}

\author{
G. Bradley Schaefer, $M D^{1}$, Nancy J. Mendelsohn, $M D^{2}$, and the Professional Practice and Guidelines Committee
}

Key Words: pervasive developmental disorders, tiered evaluations, diagnostic yield, Asperger syndrome

\begin{abstract}
Disclaimer: This guideline is designed primarily as an educational resource for medical geneticists and other health care providers to help them provide quality medical genetics services. Adherence to this guideline does not necessarily assure a successful medical outcome. This guideline should not be considered inclusive of all proper procedures and tests or exclusive of other procedures and tests that are reasonably directed to obtaining the same results. In determining the propriety of any specific procedure or test, the geneticist should apply his or her own professional judgment to the specific clinical circumstances presented by the individual patient or specimen.
\end{abstract}

\begin{abstract}
The autism spectrum disorders are a collection of conditions, which have, in common, impaired socialization and communication in association with stereotypic behaviors. The reported incidence of autism spectrum disorders has increased markedly over the past decade. In addition, a large amount of attention has been paid to these conditions among lay and professional groups. These influences have resulted in a marked increase in the number of referrals to clinical geneticists for evaluation of persons with autism spectrum disorders. The primary role of the geneticist in this process is to define etiology, if possible, and to provide counseling and contribute to case management based on the results of such investigations. In deciding upon the appropriate evaluation scheme for a particular patient, the geneticist must consider a host of different factors. Such considerations would include (1) Assuring an accurate diagnosis of autism before proceeding with any investigation. (2) Discussing testing options, diagnostic yields, and patient investment before proceeding with an evaluation. (3) Communication and coordination with the patient's medical home. (4) Assessing the continuously expanding and evolving list of available laboratory testing modalities in light of evidence-based medicine. (5) Recognizing expanded phenotypes of well-described syndromic and metabolic conditions that encompass autism spectrum disorders. (6) Defining an individualized evaluation scheme based on the unique history and clinical features of a given patient. The guidelines in this article have been developed to assist the clinician in the consideration of these factors. Genet
\end{abstract} Med 2008:10(4):301-305.

Autism spectrum disorders (ASDs), also known as pervasive developmental disorders (PDD), are a behaviorally defined group of neurodevelopmental disorders that are usually diagnosed in early childhood. They are characterized by varying degrees of restrictions in communication and social interaction and by atypical, repetitive behaviors. The phenotype of ASDs is extremely heterogeneous with differences from person

\footnotetext{
From the ${ }^{I}$ University of Nebraska Medical Center, Munroe-Meyer Institute for Genetics and Rehabilitation, Omaha, Nebraska; and ${ }^{2}$ Division of Medical Genetics, Children's Hospitals and Clinics of Minnesota, Minneapolis, Minnesota.

G. Bradley Schaefer, MD, 985430 Nebraska Medical Center, Omaha, NE 68198-5430. Email: gbschaef@unmc.edu.

Go to www.geneticsinmedicine.org for a printable copy of this document.

Submitted for publication January 28, 2008.

Accepted for publication January 29, 2008.

DOI: $10.1097 / G I M .0 b 013 e 31816 b 5 c c 9$
}

to person in a wide range of symptoms and severity as well as differences between the various subtypes of ASDs (e.g., autistic disorder, Asperger, PDD [NOS]).

Multiple lines of epidemiologic evidence support the strong role of genetics in the etiology of ASDs. ${ }^{1-3}$ Results of population studies of unselected cases of autism are consistent with multifactorial inheritance. The reported recurrence risk for full siblings is $4 \%$ if the affected child is a girl and $7 \%$ if the affected child is a boy. Overall, $2-3 \%$ of families have more than one affected child (potentially because of a decreased occurrence of subsequent pregnancies). If a second child has autism, the recurrence risk is on the order of $25-35 \%$. The calculated relative recurrence risks are 22.3 for autism and 13.4 for Asperger syndrome. The sibling risk ratio $\left(\lambda_{\mathrm{s}}\right)$ is estimated to be $100-150$. The calculated heritability (the proportion of phenotypic variance explained by the genotype) is around $90 \%$. There is an excess of twins reported in affected sib pairs. Population stud- 
ies show a concordance of $70 \%$ in monozygotic twins; $90 \%$ if the broader phenotypic definition is used. This is in contrast to a $3 \%$ concordance in dizygotic twins. ${ }^{2-5}$

As a group, ASDs occur three to four times more commonly in men. Such a sexual dimorphism suggests that X-linked genes play a major role in the etiology of the spectrum. However, whole genome screens have found only four minor linkages to the $\mathrm{X}$ chromosome, and $\mathrm{X}$ chromosome genes seem to account for a only a small portion of the overall genetic contribution. Evidence of linkage has been found to most autosomes, suggesting marked genetic heterogeneity. The most consistently reported linkages have been with chromosomal locations 15q11-13, 7q 22-31 (two loci with parent of origin effect), 13q, 17q 11 (male-specific locus), 2q, and 16p. ${ }^{6-11}$

Over the past decade, the reported incidence of ASDs has increased markedly with some estimates suggesting a quadrupling in 10 years. The current estimates for autism are now reported to be on the order of 10-60 per 10,000 individuals, if all forms of ASDs are considered. In fact, the Center for Disease Control and Prevention has recently estimated the prevalence of ASDs in the United States at approximately 5.6 per 1000 (1 of 155 to 1 of 160) children. ${ }^{12,13}$ This rise in the reported prevalence of ASDs is unlikely to represent a true "epidemic" of the condition as has been suggested by some. Rather, it seems that, this reported increase can be attributed to better knowledge of the disease and its variability, broader diagnostic criteria, improved public and professional awareness, and a higher level of acceptance of the diagnosis.

The role of the clinical geneticist is to determine the etiology of the ASDs, if possible, and to provide counseling for the family. In recent years, there has been an explosion of new diagnostic options and tools available to the clinician. Several recent publications have also reported a host of "expanded phenotypes" for genetic and metabolic conditions in association with ASDs phenotypes. ${ }^{14-19}$ These factors have led to an increase in the number of referrals to the clinical geneticist and an increase in the diagnostic yield. Now, more than ever, medical genetics services are available to help families answer the question "Why?"

In deciding upon an evaluation plan, the clinical geneticist has the difficult task of balancing an ever-expanding list of available tests and possible diagnoses with the issues of cost, practicality, and expected yield. The guidelines put forth here outline a strategy of a tiered evaluation of the etiology of autism. These recommendations use evidence-based conclusions from the current available literature and cumulative clinical experience.

\section{RATIONALE FOR AN EVALUATION}

The rationale for a clinical genetics evaluation for persons with ASDs has been questioned by some. Concerns have been expressed over the high cost of such an evaluation coupled with the fact that the information obtained typically will not change interventions for the patient. The rationale for performing a clinical genetics consultation for a patient with an ASD is clear to the clinical geneticist. Clinical geneticists can contribute to the process by examining and evaluating the patient, the parents, and siblings, as necessary, in establishing the etiology. A definitive diagnosis helps the patient acquire needed services, and is helpful in many other ways for the family. Many families are greatly empowered by knowledge of the underlying cause of a relative's disorder. Depending on the etiology, associated medical risks may be identified that lead to screening and the potential for prevention of morbidity. Specific recurrence risk counseling-beyond general multifactorial informationcan be provided, and targeted testing of at risk family members can be offered. In a limited number of cases (e.g., metabolic disorders) targeted therapies may be or become available. These significant positive benefits strongly justify a medical genetics consultation for all patients with ASDs. One of the best strategies for integrating clinical genetics services into the care of patients with ASDs can be the participation of the geneticist on an interdisciplinary "autism team." This allows the geneticist to work alongside other professionals involved in the care of persons with ASDs with access to detailed, specific diagnostic information about the patient.

\section{REPORTED APPROACHES AND YIELDS}

The generally reported rate of success for identifying a specific (unifactorial) diagnosis in persons with autism is $6-15 \%{ }^{20-22}$ This range is applicable even for evaluations of patients with PDD (NOS), atypical autism, Asperger syndrome, or autistic features, which did not necessarily meet the Diagnostic and Statistical Manual of Mental Disorders, 4th Edition, criteria for PDD. Many factors seem to influence the diagnostic yield. The use of newer diagnostic modalities and the aggressiveness of the evaluation seem to be the most critical. Not surprisingly, the skill and experience of the geneticist also factor heavily into the yield.

A critical review of the potential contribution of newer testing techniques suggests that yield can be significantly higher than $15 \%$. Chromosomal studies are consistently reported as giving one of the highest diagnostic yields in persons with ASDs. ${ }^{23-25}$ Continued improvements in cytogenetic approaches including higher resolution studies have further increased the diagnostic yield.

Numerous submicroscopic deletions and duplications have been reported in association with an autism phenotype. In general, the most commonly reported loci mirror the reported linkage data. Some of the most frequently reported regions with abnormalities in association with ASDs include 15q pericentromeric $^{11-13}$ region, 17p11, 22q11, 22q13, and 2q37. Most recently, changes in the $16 \mathrm{p} 11.2$ region have been reported as occurring in a significantly high frequency in patients with ASDs - prompting the designation of this region as a "hot spot of genetic instability." $26-28$

Currently, array comparative genomic hybridization (aCGH) has emerged as a powerful new tool that promises further revolution of clinical genetic testing. The technology of assessing submicroscopic rearrangements is evolving at a mind-bog- 
gling rate. New platforms are being developed at rates faster than clinical studies can define their use. The availability of multiple platforms further complicates the ability to compare studies from various sites. Relatively few studies have been published that provide an actual estimate of the diagnostic yield of aCGH in evaluating patients with autism. One study found a $27.5 \%$ yield in the study of aCGH in patients with "syndromic" autism. ${ }^{29}$ Preliminary data from many sites suggests that the cumulative yield of aCGH will prove to be the highest yield test that is clinically available. If the estimates of the frequency of the most commonly reported anomalies are pooled, current aCGH platforms can be estimated to identify abnormalities on the order of $10 \%$, beyond what would be identified by standard chromosomal testing (G. Schaefer, unpublished data). Until definitive, large-scale studies provide confirmation of the use of aCGH, its role in the evaluation of ASDs may not be fully appreciated. Realistic predictions suggest that the time in which this will occur may be just a few years.

The strong association of autism with Fragile $\mathrm{X}$ syndrome has been confirmed in almost every large reported series. ${ }^{15,30,31}$ Mutations in the Methyl-CPG-Binding Protein 2 (MECP2) gene are reported in a significant number of women with autism. ${ }^{20,32}$ To date, no male with idiopathic autism has been reported with a mutation in the MECP2 gene. Mutations in the phosphatase and tensin homolog (PTEN) gene are reported to occur frequently in the subgroup of patients with autism with a head circumference 2.5 SDs greater than age-appropriate means. ${ }^{14}$ Clinically recognizable syndromes and metabolic disorders are other identifiable causes. ${ }^{33}$

A synthesis of the published literature suggests that the following diagnostic yields would be projected in the genetic evaluation of ASDs:

- High-resolution chromosome studies (5\%)

- aCGH-beyond what would be detected by chromosomal analysis $(10 \%)$

- Fragile X $(5 \%)$

- MECP2 (5\%-women only)

- $\operatorname{PTEN}(3 \%$-if head circumference $>2.5 \mathrm{SDs})$

- Other (10\%)

Thus, using current knowledge and technology, a thorough clinical genetics evaluation of persons with ASDs will result in a positive answer in up to $40 \%$ of individuals.

\section{EVALUATION SCHEME}

The first (and most critical) step in the clinical genetic evaluation of ASDs is the pre-evaluation. Several pieces of critical information need to be obtained before beginning any investigation. An accurate ASD diagnosis is mandatory. The diagnosis of ASDs should be made by appropriately trained professionals using objective criteria. Normal hearing should be documented because children with significant hearing loss tend to have difficulties with socialization and communication that may be misidentified as autism. Recently published guide- lines from the American Academy of Pediatrics suggest that primary care providers obtain chromosome and Fragile X studies at the time an ASD is diagnosed. ${ }^{34}$ Thus, part of the evaluation may already have been accomplished before referral to the geneticist.

As with all clinical evaluations, an etiologic evaluation must be tailored to the individual patient. The design of the evaluation must take into consideration focused information from the history and physical as well as clinical experience. There is no single approach or algorithm that can be applied to all cases. For practical reasons, a step-wise (tiered) evaluation is considered by many to be the preferred approach. In general, a nontiered evaluation in which a large battery of tests is ordered as part of the initial testing scheme is poorly tolerated by the patient and family and less acceptable to third-party payers. A stepwise evaluation can be designed such that tests obtained in higher (earlier) tiers have a greater expected diagnostic yield, lower invasiveness of testing, better potential of intervention, and easier overall practicality. ${ }^{33} \mathrm{~A}$ model for such a tiered evaluation is provided in Table 1 .

This scheme will evolve with continued advancements in diagnostic testing and improved understanding of the ASD phenotype. Additional conditions have already been reported in association with an ASD phenotype, but to date none of these have been evaluated in a large prospective cohort. Thus, the possibility of a fourth (extended) tier of evaluation is a distinct possibility in the near future. Alternatively, advances in technology may permit bundling of individual tests into an extended, more readily accessible, and lessexpensive platform.

\section{RECOMMENDATIONS}

1. Accurate diagnosis: It is critically important that a firm diagnosis of ASD is made before initiating any genetic evaluation. Although the diagnosis of autism may seem straightforward, many neurodevelopmental disorders have overlapping phenotypes. The diagnosis should be made by a professional trained in the diagnosis of autism. The patient's parents, siblings, and offspring may also need to be evaluated. Objective criteria with the application of generally accepted tools should be used. All persons with apparent autism should have a formal audiogram to rule out a significant hearing loss.

2. Role of the primary care physician: All persons with autism should have a designated primary care physician (PCP). Often the PCP will be the first professional to raise the question of ASD as a possible diagnosis. Depending on training and comfort level, the PCP may be prepared to make a diagnosis of an ASD. Alternatively, the PCP may make a referral to a school team or mental health professional for diagnostic confirmation. Recent guidelines from the American Academy of Pediatrics suggest that the PCP obtain high-resolution chromosome studies (peripheral karyotype) and Fragile X studies when the diagnosis of an ASD is confirmed. After clinical genetics 
Table 1

Template for the clinical genetic diagnostic evaluation of autism spectrum disorders
Pre-evaluation

Confirmation of diagnosis of autism by trained professional using objective criteria and tools

Sensory screening (complete audiogram)

Electroencephalogram—if clinical suspicion of seizures

Cognitive testing

Verify results of newborn screening

[High-resolution chromosomal analysis and Fragile X studies may be performed before referral]

First tier

Initial evaluation to identify known syndromes or associated conditions

Examination with special attention to dysmorphic features

Should include Woods lamp evaluation

If specific diagnosis is suspected, proceed with targeted testing

Rubella titers-if clinical indicators present

"Standard" metabolic screening_if clinical indicators present and if suspected condition was not assessed by newborn screening

Urine mucopolysaccharides and organic acids

Serum lactate, amino acids, ammonia, and acyl-carnitine profile

High-resolution chromosomal analysis—if not already performed

DNA for Fragile X-if not already performed

Second tier

Fibroblast karyotype if leukocyte karyotype is normal and clonal pigmentary abnormalities are noted

Comparative genomic hybridization (chromosomal microarray) ${ }^{a}$

$M E C P 2$ gene testing (females only)

PTEN gene testing (if the head circumference is $2.5 \mathrm{SD}$ greater than the mean)

Third tier

Brain magnetic resonance imaging

Serum and urine uric acid

If elevated, Hypoxanthine-guanine phosphoribosyl transferase ( $H g P R T)$ and Phosphoribosylpyrophosphate (PRPP) synthetase superactivity testing

If low, purine/pyrimidine panel (uracil excretion, xanthine, hypoxanthine)

Extracted from Schaefer GB and Mendelsohn NJ. Genetics evaluation for the etiologic diagnosis of autism spectrum disorders. Genet Med 2008;10:4-12.

${ }^{a}$ Advances in microarray technology will likely elevate aCGH to a first tier study in the near future.

consultation, the PCP and the clinical geneticist should be prepared to partner in ordering, scheduling, and coordination of recommended diagnostic testing.

3. Referral for clinical genetics evaluation: Defining the etiology of an ASD can be of great benefit to the patient and family. Information gained from an identified etiology can help with family counseling, medical management, preventative health strategies, and empowerment of the family. Clinical geneticists have much to offer in this process beyond the initial assessments made by the PCP or mental health professionals working with the individual or family with ASD. As such, a genetic consultation should be offered to all persons and families with ASDs. Evaluations should be considered for any individual along the full autism spectrum. The referring professional should discuss expectations and possible outcomes of such an evaluation before making the referral. The referring professional should be aware of what is involved in such a consultation and the potential diagnostic yields and share this information with the patient and family.

4. Tiered evaluation: The clinical genetic evaluation of an individual with an ASD must be customized to the clinical situation. A patient may be referred to the geneticist with a specific diagnosis that is being considered-seeking confirmation. Alternatively, a syndromic diagnosis may be apparent to the geneticist upon the initial visit. In either case, the diagnosis should be confirmed using accepted clinical criteria and laboratory testing (if available). Many recognizable syndromes have a firmly documented association with autism. For these conditions, further investigation into the etiology of the ASD is unnecessary. There are, however, genetic conditions that have been reported in association with ASDs in which the reported association is not as convincing. For patients with these conditions, it is recommended that an etiologic evaluation for the ASD proceed as an independent condition. Table 2 provides a partial list of these two groups of conditions. If the clinical geneticist does not identify a specific disorder upon the initial evaluation, further testing can be accomplished as outlined in Table 1.

5. Counseling: Upon completion of the clinical genetics evaluation, two groups of individuals will have been identified: those with and those without an identifiable major single etiology. Definitive counseling should be provided to both groups. For those without an identifiable etiology, counseling should be provided for multifactorial inheritance. The best available published empiric recurrence risks for full siblings are $4 \%$ if the affected child is a girl and 7\% if the affected child is a boy. If a second child has autism, the reported recurrence risk has been from $25 \%$ to $50 \%$. A reasonable synthesis of published reports would be around $30 \%$.

6. Follow-up: Clinical geneticists differ greatly in their degree of involvement with patients after completion of diagnostic consultations. Intervening changes in technology and in phenotypes often aid in ultimately obtaining a diagnosis. At a minimum, periodic reevaluations should be considered for patients in whom a definitive etiology is not initially discovered. The timing of interval follow-ups should be a negotiation between the patient and family, the PCP, and the geneticist. 
Table 2

Partial list of genetic syndromes with a reported association with autism

\begin{tabular}{ll}
\hline No work-up indicated & Autism evaluation indicated \\
\hline Fragile X syndrome & Apert syndrome \\
Rett syndrome & Williams syndrome \\
Angelman syndrome & Joubert syndrome \\
Prader-Willi syndrome & Noonan syndrome \\
Smith-Lemli-Opitz syndrome & Down syndrome \\
Smith-Magenis syndrome & Turner syndrome \\
Tuberous sclerosis & Neurofibromatosis \\
PTEN associated disorders & Myotonic dystrophy, \\
Rowden syndrome, Bannayan- & Duchenne dystrophy \\
Shprintzen syndrome & \\
(22q11 deletions) & Moebius anomalad \\
Sotos syndrome & Cohen syndrome \\
CHARGE syndrome & Oculo-auriculo-vertebral \\
& spectrum
\end{tabular}

Hypomelanosis of Ito

Lujan-Fryns syndrome

De Lange syndrome

Extracted from Schaefer GB and Mendelsohn NJ. Genetics evaluation for the etiologic diagnosis of autism spectrum disorders. Genet Med 2008;10:4-12.

\section{RESOURCES}

Autistic spectrum disorders: best practice guidelines for screening, diagnosis, and assessment. Sacramento, CA: California Department of Developmental Services, 2002.

Autism Genetics Resource Exchange. Available at: http:// www.agre.org/

Autism Society of America. Available at: http://www. autism-society.org/site/PageServer

National Institute of Mental Health. Autism spectrum disorders (pervasive developmental disorders). Available at: http://www.nimh.nih.gov/health/publications/autism/ complete-publication.shtml

\section{References}

1. Chudley AE. Genetic landmarks through philately - autism spectrum disorders: a genetic update. Clin Genet 2004;65:352-357.

2. Cook EH Jr. Genetics of autism. Child Adolesc Psychiatr Clin N Am 2001;10:333-350.

3. Muhle R, Trentacoste SV, Rapin I. The genetics of autism. Pediatrics 2004;113:e472e486.

4. Lotspeich LJ, Ciaranello RD. The neurobiology and genetics of infantile autism. Int Rev Neurobiol 1993;35:87-129.

5. Spence SJ. The genetics of autism. Semin Pediatr Neurol 2004;11:196-204.

6. Cook E Jr, Courchesne RY, Cox NJ, Lord C, et al. Linkage-disequilibrium mapping of autistic disorder, with 15q11-13 markers. Am J Hum Genet 1998;62:1077-1083.

7. Falk RE, Casas KA. Chromosome $2 \mathrm{q} 37$ deletion: clinical and molecular aspects. Am J
Med Genet C Semin Med Genet 2007;145:357-371.

8. Collaborative Linkage Study of Autism. An autosomal genomic screen for autism. Am J Med Genet 2001;105:609-615.

9. Goizet C, Excoffier E, Taine L, Taupiac E, et al. Case with autistic syndrome and chromosome 22q13.3 deletion detected by FISH. Am J Med Genet 2000;96:839-844.

10. Nakamine A, Ouchanov L, Jimenez P, Manghi ER, et al. Duplication of 17 (p11.2p11.2) in a male child with autism and severe language delay. Am J Med Genet A 2008;146:636-643.

11. Yonan AL, Alarcon M, Cheng R, Magnusson PK, et al. A genomewide screen of 345 families for autism-susceptibility loci. Am J Hum Genet 2003;73:886-897.

12. Fombonne E. The prevalence of autism. JAMA 2003;289:87-89.

13. Mental Health in the United States: parental report of diagnosed autism in children aged 4-17 years-United States, 2003-2004. MMWR Morbid Mortal Wkly Rep 2006; 55:481-486.

14. Butler MG, Dasouki MJ, Zhou XP, Talebizadeh Z, et al. Subset of individuals with autism spectrum disorders and extreme macrocephaly associated with germline PTEN tumour suppressor gene mutations. J Med Genet 2005;42:318-321.

15. Clifford S, Dissanayake C, Bui QM, Huggins R, et al. Autism spectrum phenotype in males and females with fragile X full mutation and premutation. JAutism Dev Disord 2007;37:738-747.

16. Erlandson A, Hagberg B. MECP2 abnormality phenotypes: clinicopathologic area with broad variability. J Child Neurol 2005;20:727-732.

17. Morrow JD, Whitman BY, Accardo PJ. Autistic disorder in Sotos syndrome: a case report. Eur J Pediatr 1990;149:567-569.

18. Park JP, Moeschler JB, Davies WS, Patel PI, et al. Smith-Magenis syndrome resulting from a de novo direct insertion of proximal 17q into 17p11.2. Am J Med Genet 1998;77:23-27.

19. Pearl PL, Gibson KM, Acosta MT, Vezina LG, et al. Clinical spectrum of succinic semialdehyde dehydrogenase deficiency. Neurology 2003;60:1413-1417.

20. Abdul-Rahman OA, Hudgins L. The diagnostic utility of a genetics evaluation in children with pervasive developmental disorders. Genet Med 2006;8:50-54.

21. Battaglia A, Carey JC. Etiologic yield of autistic spectrum disorders: a prospective study. Am J Med Genet C Semin Med Genet 2006;142:3-7.

22. Schaefer GB, Lutz RE. Diagnostic yield in the clinical genetic evaluation of autism spectrum disorders. Genet Med 2006;8:549-556.

23. Reddy KS. Cytogenetic abnormalities and fragile-X syndrome in autism spectrum disorder. BMC Med Genet 2005;6:3.

24. Shevell MI, Majnemer A, Rosenbaum P, Abrahamowicz M. Etiologic yield of autistic spectrum disorders: a prospective study. J Child Neurol 2001;16:509-512.

25. Weidmer-Mikhail E, Sheldon S, Ghaziuddin M. Chromosomes in autism and related pervasive developmental disorders: a cytogenetic study. I Intellect Disabil Res 1998;42(pt 1):8-12.

26. Weiss LA, Shen Y, Korn JM, Arking DE, et al. Association between microdeletion and microduplication at 16p11.2 and autism. N Engl J Med 2008;358:667-675.

27. Kumar RA, Karamohamed S, Sudi J, Conrad DF, et al. Recurrent 16p11.2 microdeletions in autism. Hum Mol Genet 2008;17:628-638.

28. Ullmann R, Turner G, Kirchhoff M, Chen W, et al. Array CGH identifies reciprocal $16 \mathrm{p} 13.1$ duplications and deletions that predispose to autism and/or mental retardation. Hum Mutat 2007;28:674-682.

29. Jacquemont ML, Sanlaville D, Redon R, Raoul O, et al. Array-based comparative genomic hybridisation identifies high frequency of cryptic chromosomal rearrangements in patients with syndromic autism spectrum disorders. J Med Genet 2006;43: 843-849.

30. Hatton DD, Sideris J, Skinner M, Mankowski J, et al. Autistic behavior in children with fragile X syndrome: prevalence, stability, and the impact of FMRP. Am J Med Genet A 2006;140:1804-1813.

31. Watson MS, Leckman JF, Annex B, Breg WR, et al. Fragile X in a survey of 75 autistic males. N Engl J Med 1984;310:1462.

32. Carney RM, Wolpert CM, Ravan SA, Shahbazian M, et al. Identification of MeCP2 mutations in a series of females with autistic disorder. Pediatr Neurol 2003;28:205211.

33. Schaefer GB, Mendelsohn NJ. Genetics evaluation for the etiologic diagnosis of autism spectrum disorders. Genet Med 2008;10:4-12.

34. Johnson CP, Myers SM. American Academy of Pediatrics Council on Children with Disabilities. Identification and evaluation of children with autism spectrum disorders. Pediatrics 2007;120:1183-1215. 